\title{
METALOGENIA DE ORO Y COBRE EN AMÉRICA CENTRAL
}

\author{
Carl E. Nelson ${ }^{1} \&$ Fernando Nietzen ${ }^{2}$
}

(1) 2360 23rd. Street, Boulder, Colorado 80304 USA, (303) 939-9517; E-mail: cnelson945@aol.com

(2) 150 North Terra Cotta Road,Lake Elsinore, California 92530 USA, (909) 674-9858

(Recibido 4/11/1998; Aceptado 9/8/1999)

\begin{abstract}
Gold and copper deposits in Central America are hosted by island arc volcanic rocks of Late Cretaceous and younger age. Low potassium tholeiitic basalts form the base of the volcanic pile and were deposited in an intraoceanic island arc. Volcanism became more potassic with time, and by Late Miocene time, calcalkaline andesites dominate the volcanic rock record. Alkaline volcanic rocks appeared about five million years ago but are underreported in older portions of the arc.

Porphyry copper deposits are centered on calc-alkaline plutons of intermediate (quartz diorite to quartz monzonite) composition and are typically anomalous in gold. Although some of the porphyry deposits contain in excess of a billion tonnes, none are in production. Bonanza gold veins cluster around sub-volcanic intrusions in districts which mimic the spacing of active volcanoes. Precious metal bonanzas, a number of which produced in excess of a billion ounces of gold, are responsible for the bulk of historic gold production in Central America. Hot spring gold deposits are being discovered along a regional unconformity which marks the emergence of the present-day island arc from the ocean. Although discovered only recently, some of these deposits already exceed a million ounces. Host rocks include exogenous domes and surrounding pyroclastic aprons.

Exploration efforts today focus on bulk-mineable deposits: porphyry-related stockworks, skarns, and epithermal stockworks and disseminated deposits. Regional strike-slip faults parallel to the Nicaraguan depression control the distribution of both gold and copper throughout Central America. Age dates provide documentation for mineralization throughout the history of the arc, a period of close to 100 million years.
\end{abstract}

RESUMEN: Los depósitos de oro y cobre en América Central se encuentran dentro de rocas del Cretácico Tardío y más jóvenes del arco insular volcánico. Basaltos toleíticos bajos en potasio forman la base de la agrupación volcánica y fueron depositados en un arco insular intraoceánico. El vulcanismo se tornó mas potásico con el tiempo, y ya para el Mioceno tardío, las andesitas calco-alcalinas dominan la secuencia volcánica. Rocas volcánicas alcalinas aparecieron hace unos cinco millones de años pero han sido sub-reportadas en areas más antiguas del arco.

Depósitos porfídicos de cobre se centran sobre plutones calco-alcalinos de composición intermedia, diorita cuarcífera a diorita monzonítica y que típicamente son anómalas en oro. Aunque algunos de los depósitos porfídicos contienen más del billón de toneladas, ninguno está en producción. Vetas auríferas tipo bonanza se agrupan alrededor de intrusiones subvolcánicas en distritos que asemejan la distribución espacial de volcanes activos. Bonanzas de metales preciosos, algunos de los cuales produjeron por encima del millón de onzas de oro, son responsables por la mayor parte de la producción histórica en América Central. Se han venido descubriendo depósitos auríferos a lo largo de una inconformidad regional que marca el nacimiento del arco insular actual del océano. A pesar de su reciente descubrimiento, algunos de estos depósitos ya exceden el millón de onzas. Las rocas caja incluyen domos exógenos y los mantos piroclásticos que los encierran.

Los esfuerzos exploratórios de hoy se concentran sobre depósitos de alto volumen: stockworks relacionados con los pórfidos, depósitos de skarn, stockworks epitermales y depósitos diseminados. Fallas longitudinales regionales paralelas a la Depresión de Nicaragua controlan la distribución, tanto del oro como del cobre a través de Centroamérica. Dataciones proveen documentación para mineralización a lo largo de la historia del arco, un período de cerca de 100 millones de años. 


\section{INTRODUCCIÓN}

América Central continúa rezagada con respecto al resto de la Cordillera Americana en términos de producción mineral. Esta realidad es el resultado de inversión limitada en vez de riqueza mineral limitada. Durante el último medio siglo, Centroamérica ha estado caracterizado por un alto riesgo político, burocracia, y códigos mineros diseñados para mantener el control soberano de la riqueza mineral. La producción aurífera anual nunca excedió las 200000 onzas y aunque programas regionales de exploración resultaron en varios descubrimientos de pórfidos de cobre, ninguno entró en producción.

Recientes mejoras, tanto en el ambiente político como en el económico de Centroamérica están atrayendo la atención de la comunidad inversionista internacional. El producto interno bruto en la región, con la excepción de Nicaragua, ha venido creciendo a un ritmo anual de cuatro a ocho por ciento durante la presente década. En la actualidad los extranjeros pueden ser dueños de negocios locales en un $100 \%$ y la repatriación de dividendos se ha venido facilitando gradualmente. Nuevas leyes mineras como la de Honduras de 1970 y de Costa Rica de 1982, Panamá de 1989 y Guatemala de 1985 y 1993 se están modificando para atraer al inversionista. Empresas mineras estatales se han estado desmantelando.

Consecuentemente, las inversiones en exploración han aumentado; se están efectuando nuevos descubrimientos y nuevas minas están entrando en producción. En Panamá la mina Remance ha estado en producción desde 1989 y el depósito de Santa Rosa empezó a producir oro en 1995. Cerro Quema anunció en 1994 una reserva aurífera y estudios de factibilidad están nuevamente en camino en los depósitos de cobre porfídico de Cerro Colorado y de Petaquilla. En Costa Rica, el depósito aurífero de Bellavista tiene reservas de cerca del millón de onzas, mientras que el yacimiento de Las Crucitas contiene más de dos millones de onzas. Más al norte, proyectos activos incluyen a El Dorado (El Salvador) y el yacimiento tipo skarn de oro y cobre conocido como Minas de Oro (Honduras), así como la mina aurífera San Andrés. En Nicaragua, varias minas (Limón, Bonanza) continúan produciendo en el orden de 30000 a 40000 onzas de oro por año (habiendo decaido de 100000 a 120000 onzas por año a inicios de la década de los 70). Estas cifras van a mejorar con nuevas descubrimientos como Cerro Mojón que empezó de producir oro en 1997. En cuanto a producción anterior, la nación relativamente inexplorada de $\mathrm{Ni}$ caragua es la que posee mayores atributos en riqueza mineral de Centroamérica.

Este artículo describe los yacimientos de oro filoniano y depósitos de cobre hallados en Centroamérica con énfasis en descubrimientos recientes. El artículo no da a conocer manganeso del fondo marino; níquel y aluminio laterítico; tungsteno, estaño y molibdeno pegmatítico ni oro de placer. Listas exhausitivas de minas productivas antiguas, incluyendo descripciones de muchos prospectos, aparecen en Roberts \& Irving (1957), Levy (1970) y Kesler et al. (1990). Los cuadros que acompañan a este trabajo presentan datos de reserva y ley de los yacimientos conocidos. Datos de producción de algunos yacimientos están incompletos y las reservas se basan en datos con cierta variación en su confiabilidad. A pesar de estas flaquezas, los cuadros se pueden usar para identificar los tipos de yacimientos que pueden encontrarse y hacer algunas evaluaciones de su reserva y ley potencial. Los mapas de ubicación de los depósitos se pueden emplear para concentrarse sobre distritos mineros, en vez de mineralizaciones aisladas y los mapas geológicos se pueden emplear para dirigir la exploración hacia porciones más productivas de la estratigrafía volcánica. Otros resúmenes metalogenéticos en Centroamérica están incluidos en Kesler (1978), Ferencic (1971) y Nelson (1990, 1995).

\section{GEOLOGÍA REGIONAL}

América Central está dividida en cuatro bloques corticales (Fig. 1). Hacia el norte, los bloques Maya y Chortis están compuestos por rocas ígneas y metamorficas paleozoicas, carbonatos y rocas silíceas de plataforma y rocas volcánicas - silíceas intermedias. Case et al. (1990) clasifican este material como corteza 


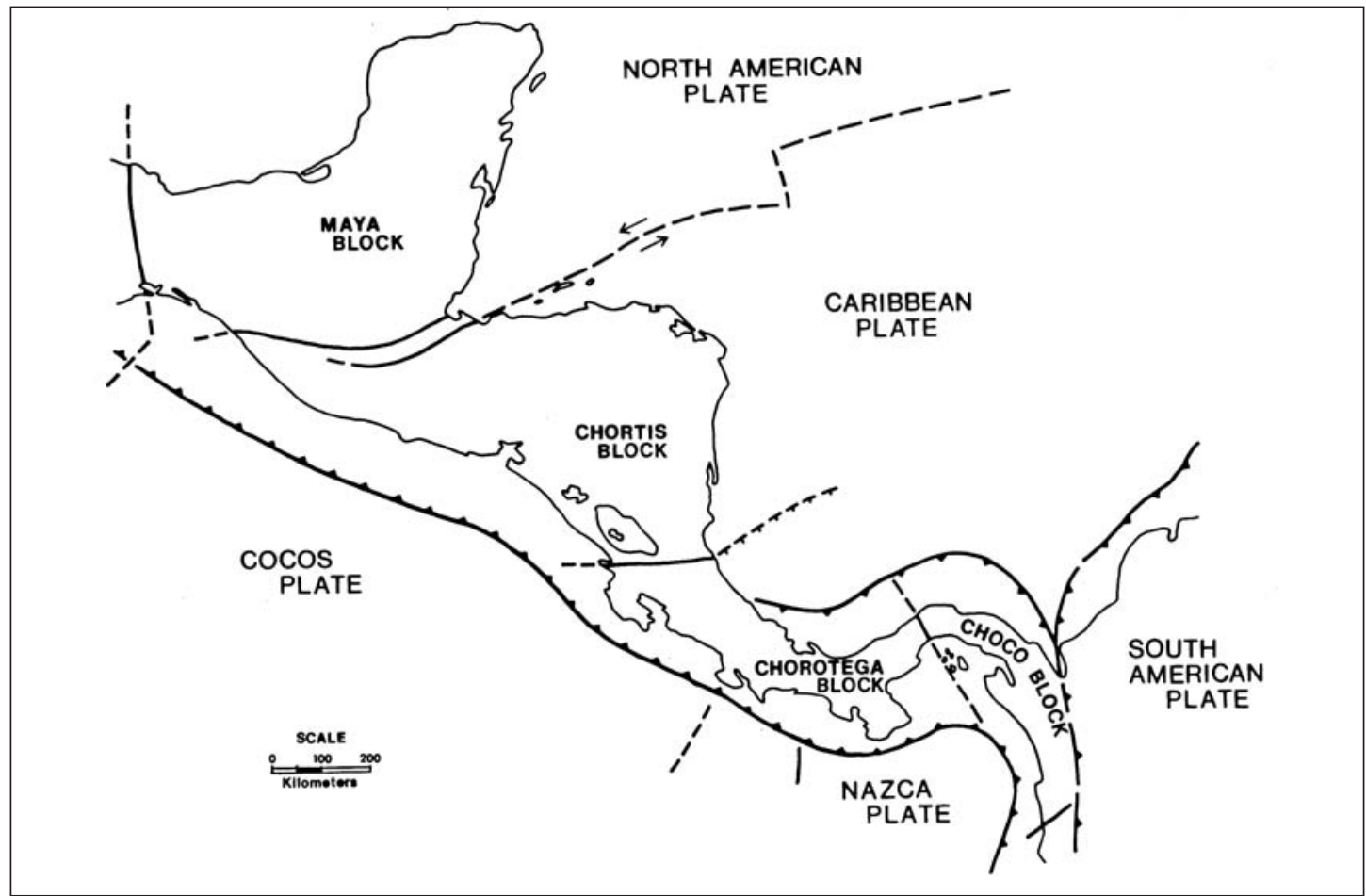

Fig. 1: Mapa tectónico regional de Centroamérica. Modificado de Donnelly et al. (1990) y Escalante (1990).

continental y transicional. Hacia el sur, los bloques Chorotega y Choco están compuestos por corteza acrecional, corteza máfica oceánica cubierta por rocas volcánicas básicas a intermedias de un arco insular. El espesor de la corteza varía desde un máximo de $45 \mathrm{~km}$ en el Bloque Chortis hasta menos de $20 \mathrm{~km}$ en el Bloque Chorotega.

Las rocas más antiguas en Centroamérica son metavolcánicas y metasedimentarias de edad paleozoica y posiblemente precámbrica. Estas rocas de la facies anfibolita están expuestas en el Bloque Maya, a lo largo de la zona de la falla Motagua, y hacia el sur a través del Bloque Chortis hasta el norte de Nicaragua. El paquete siguiente de mayor edad es una secuencia sedimentaria sin metamorfismo, depositado en cuencas intracontinentales al irse separando América del Norte y del Sur. Esta secuencia de areniscas rojizas y carbonatos de plataforma cubre grandes porciones de Guatemala, Honduras y Nicaragua.

Rocas volcánicas de edad cretácica a cuaternaria conforman el tercero y en cuanto a riqueza mineral más importante grupo de rocas. Las rocas volcánicas se pueden dividir en cuatro subgrupos incluyendo: corteza oceánica máfica y ultramáfica, basaltos del arco insular intraoceánico, ignimbritas riolíticas y andesitas calco-alcalinas, y rocas alcalinas relacionadas a la fosa tectónica. Descripciones breves de cada grupo de roca se presentan a continuación junto con cuadros de los yacimientos asociados.

Las figuras 2, 3, 4, y 5 muestran la distribución de estos grupos de rocas, la estructura regional, y la ubicación de yacimientos de oro y cobre en el la parte sur y el núcleo de Centroamérica. La información disponible sobre el tamaño de los yacimientos y su ley ha sido compilado en cuadros ligadas a los mapas e incluyen referencias a la literatura. El cuadro 1 enumera los yacimientos encajados por rocas metamorficas; el cuadro 2 contiene los depósitos tipo "skarn" y reemplazamiento; el cuadro 3 provee datos sobre depósitos porfídicos; los cuadros 4, 5 y 6 compilan información sobre depósitos de bonanza, tipo fuente termal y de alta sulfuración. 


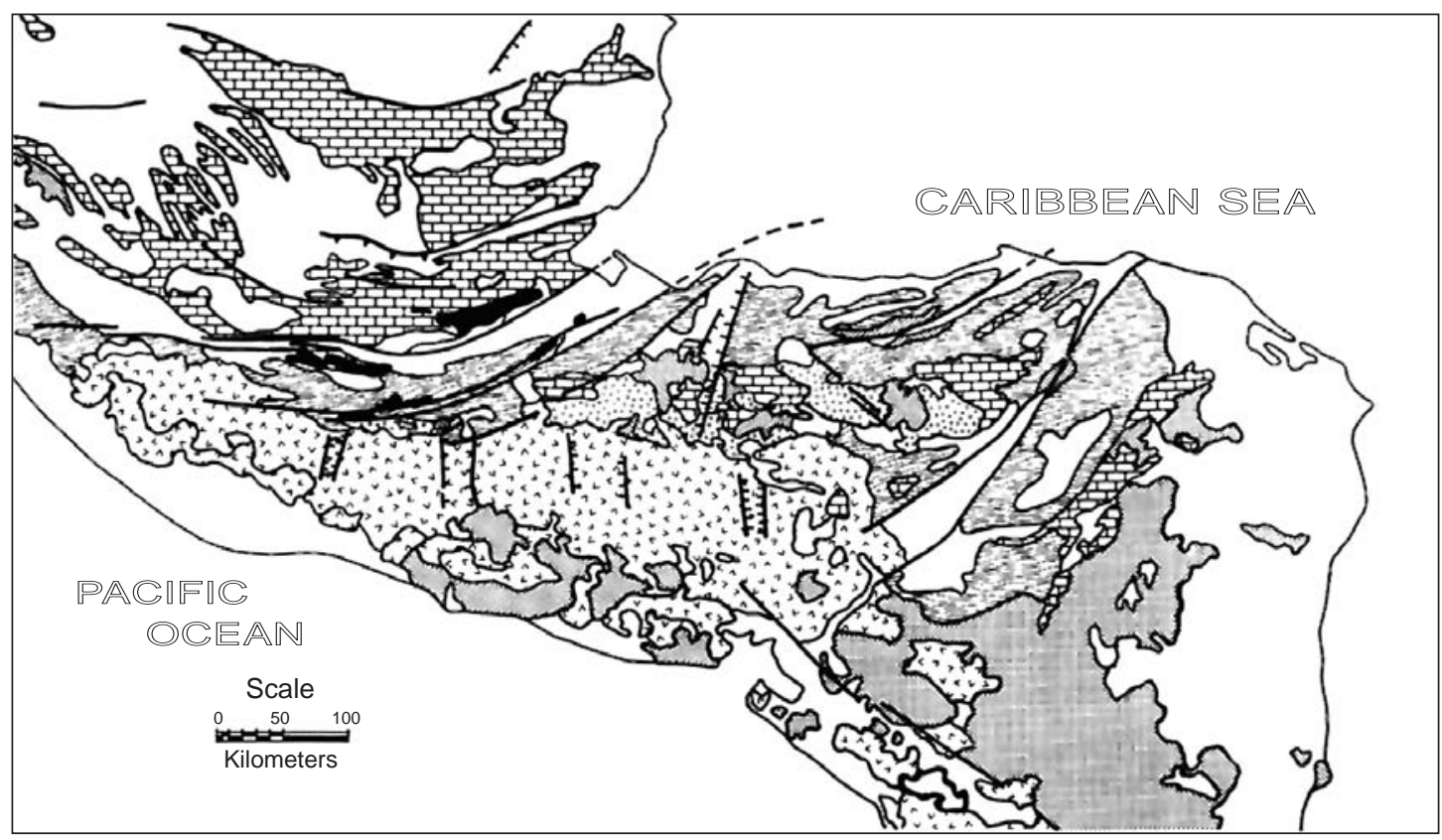

Fig. 2: Mapa geológico del núcleo de América Central. Modificado de mapas de Case \& Holcombe (1980); Kozuch (1990); Bonis, et al. (1970) y Williams \& Sanchez (1972). Las áreas con un patrón de línea curva indican que son subyacidas por rocas metamorficas; el patrón de ladrillo indica carbonatos de la plataforma; círculos abiertos indican areniscas rojizas. Las rocas ígneas se dividen en ultramáficas (negro sólido), arco insular primitivo (patrón punteado), calcoalcalinas (patrón en "v"), y alcalinas (línea horizontal segmentada).

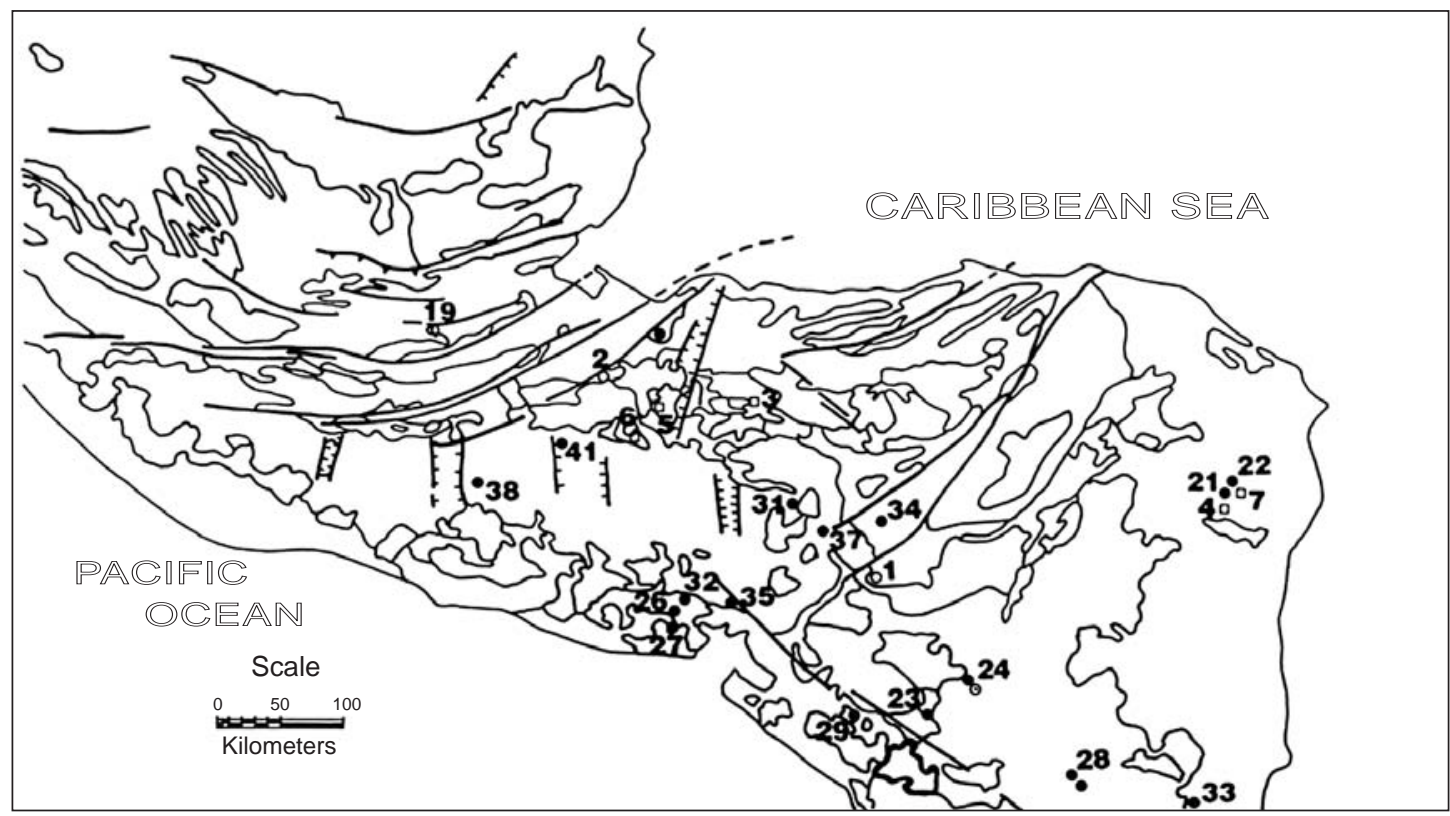

Fig. 3: Mapa de ubicación de yacimientos de oro y cobre en el núcleo de Centroamérica. Los números se refieren a los yacimientos presentados en los cuadros 1 a 6 . Los símbolos corresponden a los siguientes tipos de yacimientos: yacimientos de cobre-oro porfídicos (círculo con un punto sólido), yacimientos epitermales de oro (puntos sólidos), yacimientos de sulfuros masivos (rombos), yacimientos de skarn y reemplazamiento (cuadrados), y yacimientos de oro y cobre encajados en zonas de desgarre (círculos abiertos). 




Fig. 4: Mapa geológico del sur de Centroamérica. Modificado de mapas por Case \& Holcombe(1980), Darce (1990), Mann \& Corrigan (1990) y Tournon \& Alvarado (1997). Las rocas ígneas se dividen en rocas ultramáficas (negro sólido); piso marino (patrón con líneas de orientación al azar); y arco insular primitivo (patrón punteado), arco insular calcoalcalino (patrón en "v"), y alcalinas (línea segmentada horizontal).

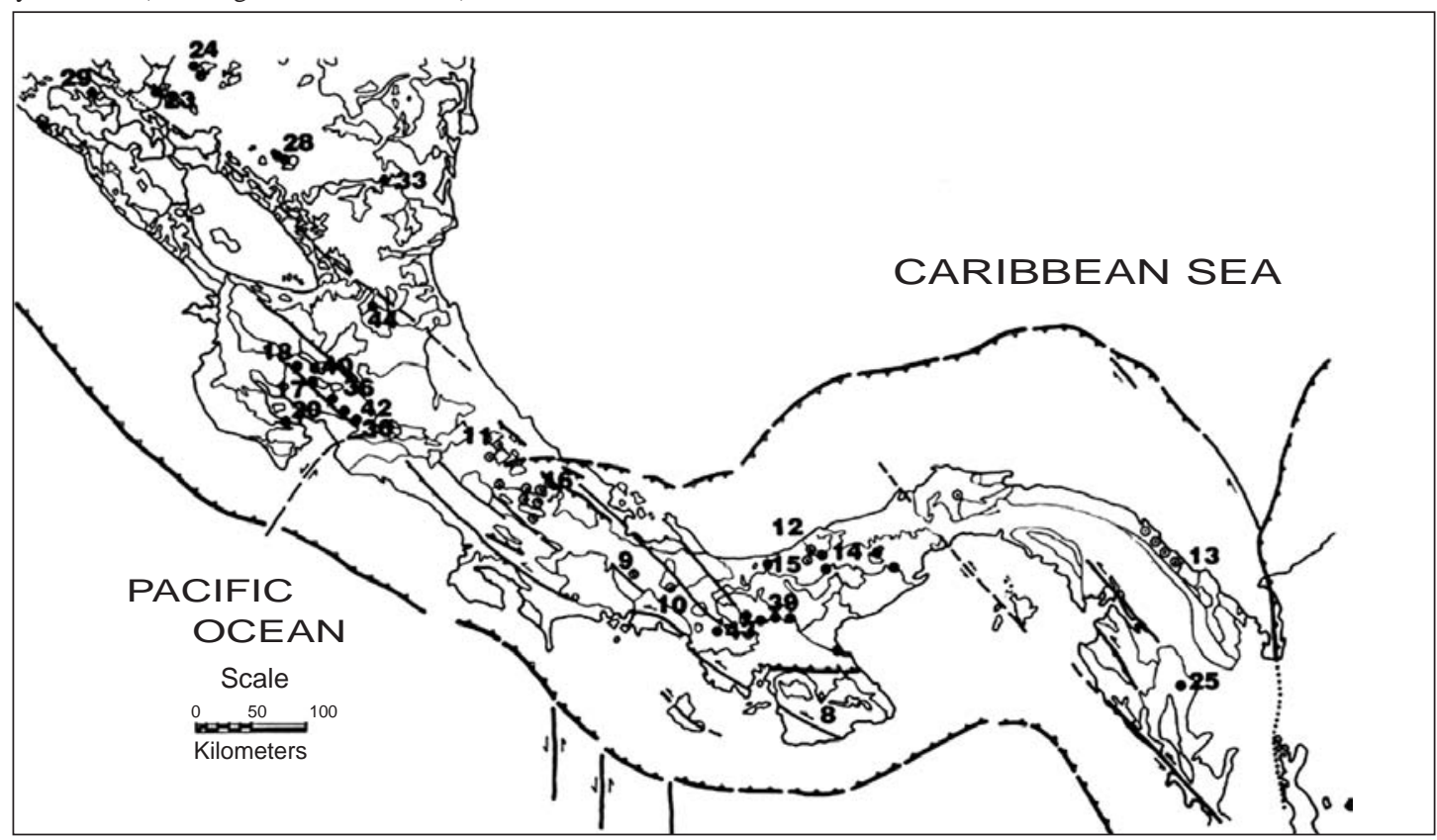

Fig. 5: Mapa de ubicación de yacimientos de oro y cobre para el sur de Centroamérica. Los números hacen referencia a los yacimientos en los cuadros 1 a 6 . Los símbolos corresponden a los siguientes tipos de yacimientos: yacimientos de oro - cobre porfídicos (círculos con un punto sólido), yacimientos epitermales de oro (puntos sólidos), yacimientos de oro y cobre de alto sulfuración (rombos). 
Cuadro 1

Yacimientos de cobre y oro en rocas metamorficas

\begin{tabular}{|c|c|c|}
\hline $\begin{array}{l}\text { Yacimiento } \\
\text { (Ubicación) }\end{array}$ & $\begin{array}{l}\text { Producción Pasada } \\
\text { (Reserva/Recurso) }\end{array}$ & Referencias \\
\hline Batolito & desconocido: numerosas & Levy, 1970 \\
\hline Dipilto & $\begin{array}{l}\text { minas de oro } \\
\text { incluyendo Alhambra, } \\
\text { Azabache }\end{array}$ & $\begin{array}{l}\text { Kesler et al, } \\
1990\end{array}$ \\
\hline $\begin{array}{l}\text { Honduras } \\
\text { Nicaragua } \\
\text { (1) }\end{array}$ & $\begin{array}{l}\text { Conchagua, Murra, } \\
\text { San Albino, } \\
\text { San Juan Telapaneca, } \\
\text { y La Virgen. }\end{array}$ & \\
\hline $\begin{array}{l}\text { Vueltas } \\
\text { del Río, } \\
\text { Honduras } \\
\text { (2) }\end{array}$ & $\begin{array}{l}466 \mathrm{~kg}(15000 \text { onzas }) \mathrm{Au} \text {. } \\
(10 \text { milliones toneladas } \\
\text { métricas a } 1,5 \mathrm{ppm} \mathrm{Au}) \\
(4,1 \text { milliones toneladas } \\
\text { métricas a } 0,67 \% \mathrm{Cu})\end{array}$ & Moe, 1992 \\
\hline
\end{tabular}

Todos los yacimientos de cobre porfídico con verificación por perforaciones han sido incluidos. Yacimientos auríferos se incluyeron si la producción o las reservas sobrepasan 3000 kg (100 000 onzas).

\section{COMPLEJO METAMÓRFICO DEL PALEOZÓICO}

Las rocas centroamericanas con datación más antigua son rocas metamorficas de la facies anfibolita de edad ordovícica hasta pérmica. Este complejo se halla mejor expuesto en Guatemala central y oriental, en donde se conoce como la Serie Chaucus así como en Honduras centrooriental donde han sido asignadas al Grupo Las Ovejas. Horne et al. (1976) dan dataciones para plutones que intruyen este complejo en un rango de hasta $305 \mathrm{Ma}$. Sobreyaciendo los grupos Las Ovejas y Chaucus están rocas con metamorfismo leve, a las que se les refiere informalmente como el Grupo Santa Rosa en Guatemala y Esquisto Cacaguapa en Honduras y el norte de Nicaragua. Una descripción detallada del complejo metamórfico Paleozóico se encuentra en Donnelly et al. (1990).

Pocos yacimientos de oro o cobre han sido descubiertos en rocas metamórficas (Cuadro 1). Unas cuantas minas pequeñas de oro y tungsteno rodean el batolito Dipilto del Cretácico tardío en Nicaragua y Honduras (Kesler et al., 1990). El yacimiento de oro y cobre Vueltas del Río en el norte de Honduras está encajado por una zona de cizalla en el basamento metamórfico cretácico. La meteorización en Vueltas del Río ha producido una zona superficial enriquecida en oro.

\section{CARBONATOS MESOZOICOS DE PLATAFORMA}

El basamento metamórfico está sobreyacido de manera inconforme por areniscas rojizas del Jurásico Superior al Cretácicio Inferior (Formación Todos Santos), calizas del Cretácico Inferior al Superior (Grupo Yojoa), y areniscas del Cretácico Superior al Terciario Inferior (Grupo Valle de Angeles). Muchos de los yacimientos en este grupo son reemplazamientos pequeños de plomo/zinc y plomo/zinc/plata. Sin embargo, la mina El Mochito en Honduras ha producido zinc, plomo, cobre y plata y ha estado en producción desde 1948.

Depósitos de cobre y oro tipo skarn son minados en las minas La Rosita y La Luz (Siuna) en Nicaragua y el depósito de Minas de Oro en Honduras está actualmente siendo evaluado. Los yacimientos de tipo skarn y de reemplazamiento se muestran en el Cuadro 2.

\section{ROCAS VOLCÁNICAS DEL CENOZOICO}

Las rocas sin metamorfismo más antiguas de Centroamérica son fragmentos del fondo oceánico Jurásico y Cretácico. Estas rocas máficas y ultramáficas llenaron la cuenca caribeña que se abría y se han preservado en la zona de la falla Motagua, como cuñas acrecionadas a la costa occidental de Centroamérica (Nicoya, penínsulas de Osa y Azuero), en el oriente de Panamá, (el Bloque Choco) y posiblemente en Nicaragua oriental (Venable, 1994). Encajan depósitos pequeños y auríferos de sulfuros masivos y pequeños depósitos de manganeso del fondo oceánico.

La metalogénesis cenozóica en Centroamérica se inicia realmente con la formación de 
Cuadro 2

Yacimientos de tipo skarn y reemplazamiento

\begin{tabular}{|c|c|c|}
\hline $\begin{array}{l}\text { Yacimiento } \\
\text { (Ubicación) }\end{array}$ & $\begin{array}{l}\text { Producción Pasada } \\
\text { (Reserva/Recurso) }\end{array}$ & Referencias \\
\hline $\begin{array}{l}\text { Minas de Oro, } \\
\text { Honduras } \\
\text { (3) }\end{array}$ & $\begin{array}{l}\text { no se conoce producción pasada, (27 millones } \\
\text { toneladas métricas con } 0,8 \% \mathrm{Cu} \text { y } \\
1,4 \mathrm{ppm} \mathrm{Au})\end{array}$ & Fischer-Watt, 1992 \\
\hline $\begin{array}{l}\text { La Luz (Siuna)*, } \\
\text { Nicaragua } \\
\text { (4) }\end{array}$ & $\begin{array}{l}45000 \mathrm{~kg}(1,45 \mathrm{M} \text { onzas }) \text { Au de } 12 \\
\text { millones toneladas }(1939-1962) \\
(8,8 \text { millones toneladas con } 2,6 \mathrm{ppm} \mathrm{Au})\end{array}$ & $\begin{array}{l}\text { Plecash \& Hopper, } 1963 \\
\text { Darce \& Tercero, } 1992\end{array}$ \\
\hline $\begin{array}{l}\text { Mochito*, } \\
\text { Honduras } \\
\text { (5) }\end{array}$ & $\begin{array}{l}750000 \text { toneladas } \mathrm{Zn}, 500000 \text { toneladas } \mathrm{Pb} \\
300000 \text { toneladas } \mathrm{Cu} \text {, más de } 3 \text { millones } \mathrm{kg} \\
(100 \mathrm{M} \text { onzas) } \mathrm{Ag} \text {. } \\
(6 \text { millones toneladas con } 8,5 \% \mathrm{Zn} \text {, } \\
4,5 \% \mathrm{~Pb}, 0,5 \% \mathrm{Cu}, 160 \mathrm{ppm} \mathrm{Ag})\end{array}$ & $\begin{array}{l}\text { Johnson, } 1993 \\
\text { UN Revolving Fund, } 1988\end{array}$ \\
\hline $\begin{array}{l}\text { Quita Gana, } \\
\text { Honduras } \\
(6)\end{array}$ & $\begin{array}{l}\text { (1,521 millones toneladas con } \\
2,17 \% \mathrm{Cu}, 2,46 \% \mathrm{Zn}, 55 \mathrm{ppm} \mathrm{Ag})\end{array}$ & UN Revolving Fund, 1988 \\
\hline $\begin{array}{l}\text { Rosita, } \\
\text { Nicaragua } \\
\text { (7) }\end{array}$ &  & $\begin{array}{l}\text { Plecash \& Hopper, } 1963 \\
\text { Darce \& Tercero, } 1992\end{array}$ \\
\hline $\begin{array}{l}\text { Concepcion Las } \\
\text { Minas*, } \\
\text { Guatemala } \\
\text { (38) }\end{array}$ & $\begin{array}{l}(2,2 \text { millones toneladas con } 0,75 \% \mathrm{Cu} \\
4,28 \% \mathrm{Zn}, 0,58 \% \mathrm{~Pb}, \text { y } 36 \mathrm{ppm} \mathrm{Ag})\end{array}$ & Machorro et al. ,1996 \\
\hline
\end{tabular}

* indica minas actualmente en producción

un arco insular intraoceánico cerca de la costa occidental de las américas durante el Cretácico tardío e inicios del Terciario. Esta secuencia volcánica incluye rocas de tipo toleítico, calco-alcalinas y alcalinas. Las tres series de magma han sido reconocidas en Costa Rica (Kussmaul et al., 1991 y Alvarado et al., 1992) donde el vulcanismo abarca un período de unos $80 \mathrm{Ma}$ (Campaniano hasta el presente). Basaltos toleíticos bajos en potasio dominan la estratigrafía volcánica desde el Cretácico tardío hasta el Mioceno (Alvarado et al., 1992), un período de unos $60 \mathrm{Ma}$. Basaltos calco-alcalinos y andesitas emergen durante el Mioceno tardío, reflejando una maduración del arco insular. Según Appel et al. (1994), el arco insular en Costa Rica no se torna realmente calcoalcalino hasta el Plioceno. Ignimbritas félsicas a lo largo de América Central son de edad pliocénica y menor.

Rocas volcánicas alcalinas se hallan en fosas con alineación norte-sur relacionadas con la progresión hacia oriente de la placa Caribe y a lo largo de fallas transcurrentes con orientación noroeste. La colisión de Panamá con Sudamérica se inició en el Mioceno temprano a mediano y resultó en una fuerza hacia el norte del Bloque de Panamá a lo largo de fallas transcurrentes orientadas al noroeste (Pindell \& Barrett, 1990). Estas fallas facilitaron el ascenso de un complejo de rocas volcánicas alcalinas poco estudiadas.

La mayor parte de la mineralización de oro y cobre en Costa Rica y a lo largo de Centroamérica está encajado por un arco mayormente toleítico más antiguo. Uno de los primeros 
Cuadro 3

Yacimientos de cobre porfídico

\begin{tabular}{|c|c|c|}
\hline $\begin{array}{l}\text { Yacimiento } \\
\text { (Ubicación) }\end{array}$ & $\begin{array}{l}\text { Producción Pasada } \\
\text { (Reserva/Recurso) }\end{array}$ & Referencias \\
\hline $\begin{array}{l}\text { Cerro Chorcha, } \\
\text { Panama } \\
\text { (9) }\end{array}$ & $\begin{array}{l}6 \text { perforaciones en } 1994 \\
20 \text { perforaciones en } 1995\end{array}$ & $\begin{array}{l}\text { Nelson, } 1995 \\
\text { Arlo, } 1995\end{array}$ \\
\hline $\begin{array}{l}\text { Cerro Colorado, } \\
\text { Panamá } \\
\text { (10) }\end{array}$ & $\begin{array}{l}\text { (1,3 billones toneladas con } 0,76 \% \mathrm{Cu} \\
0,01 \% \mathrm{Mo}, 5,1 \mathrm{ppm} \mathrm{Ag}, \text { y } 0,08 \mathrm{ppm} \mathrm{Au})\end{array}$ & $\begin{array}{l}\text { Clark, } 1982 \\
\text { Nelson, } 1995\end{array}$ \\
\hline $\begin{array}{l}\text { Nari, Costa Rica } \\
\text { (11) }\end{array}$ & (5 millones toneladas con $0,3 \% \mathrm{Cu}$ ) & MacKevett, 1980 \\
\hline $\begin{array}{l}\text { Petaquilla y } \\
\text { Botija, Panamá } \\
\text { (12) }\end{array}$ & $\begin{array}{l}\text { ( } 744 \text { millones toneladas con } 0,53 \% \mathrm{Cu}, . \\
0,014 \% \mathrm{MoS} 2,1,3 \mathrm{ppm} \mathrm{Ag}, \mathrm{y} \\
0,12 \mathrm{ppm} \mathrm{Au} \text { ) }\end{array}$ & $\begin{array}{l}\text { Adrian Res., } 1994 \\
\text { Nelson, } 1995\end{array}$ \\
\hline $\begin{array}{l}\text { Rio Pito, } \\
\text { Panamá } \\
\text { (13) }\end{array}$ & $\begin{array}{l}\text { (297 } 300 \text { toneladas con } 2,12 \mathrm{ppm} \mathrm{Au} \\
\text { y 4,04 ppm Ag) }\end{array}$ & U N Revolving Fund, 1981 \\
\hline $\begin{array}{l}\text { Molejón, } \\
\text { Panamá (14) }\end{array}$ & $\begin{array}{l}\text { (6,8 millones toneladas con 3,14 ppm } \\
\mathrm{Au} \text { y } 4,49 \mathrm{ppm} \mathrm{Ag} \text { ) }\end{array}$ & Adrian Res., 1994 \\
\hline $\begin{array}{l}\text { Palmilla, } \\
\text { Panamá (15) }\end{array}$ & $\begin{array}{l}\text { (12,7 millones toneladas con } 0,3 \% \\
\mathrm{Cu} \text { y } 1,02 \mathrm{ppm} \mathrm{Au})\end{array}$ & Adrian Res., 1994 \\
\hline $\begin{array}{l}\text { Sukut, } \\
\text { Costa Rica (16) }\end{array}$ & 4 perforaciones de Copper Range Expl. & Nelson, 1995 \\
\hline
\end{tabular}

yacimientos en formarse en este marco fue Cerro Quema, un depósito de oro y cobre con alto contenido de sulfuros ubicado en la península Azuero de Panamá. Similares rocas primitivas de arco insular intraoceánico encajan el yacimiento de Pueblo Viejo en República Dominicana (570 toneladas de oro), el yacimiento más grande de la región caribe.

En Centroamérica, el arco insular inicial está representado por unidades tales como el Grupo Aguacate en Costa Rica, el Grupo Coyol Inferior en Nicaragua, y la Formación Matagalpa en Honduras.

La distribución de rocas volcánicas en Centroamérica se presenta en las figuras 2, 3, 4 y 5 junto con la distribución de las rocas carbonáticas más antiguas de plataforma, areniscas rojizas y el basamento metamorfico. Los mapas separan el record voluminoso de rocas volcánicas centroamericanas en paquetes. A pesar de que los mapas son preliminares, muestran la distribución del piso oceánico, un arco insular intraoceánico primitivo, el arco insular calco-alcalino maduro y rocas volcánicas alcalinas relacionadas a la fosa a través de Centroamérica. Vendrán mejoras con la publicación de nuevos análisis químicos y dataciones, particularmente para aquellas áreas menos estudiadas fuera de Costa Rica. Por ejemplo, rocas volcánicas alcalinas han sido sub-reportadas a lo largo de la América Central; algunas rocas calco-alcalinas están presentes en áreas mostradas como arco insular primitivo; y áreas mapeadas como del piso oceánico Mesozoico también incluyen rocas del arco insular intraoceánico. 
Cuadro 4

Yacimientos de oro y plata tipo Bonanza

Yacimiento

(Ubicación)

\section{Distrito}

Abangares, Costa Rica

Tres Hermanos*

\author{
San Martin \\ El Recio \\ (17) \\ El Libano, Costa Rica \\ (18)
}

Bellavista, Costa Rica

(20)

Bonanza*, Nicaragua

(21)

Vesubio,

Nicaragua (22)

La India,

Nicaragua

(23)

La Reina, Nicaragua (24)

Cana,

Panamá

(25)

Divisadero,

El Salvador (26)

Hormiguero, El Salvador (27)

La Libertad*, Nicaragua (28)

Limon*,

Nicaragua

(29)

Mojón, Nicaragua (28)

\section{Producción Pasada \\ (Reserva/Recurso)}

$29000 \mathrm{~kg}$ (936 000 onzas) $\mathrm{Au}$

$18700 \mathrm{~kg}$ (600 000 onzas) Ag (1900- 1913)

$3630 \mathrm{~kg}$ (116 000 onzas) Au de

363000 toneladas métricas mena

(272 000 toneladas con 7,9 ppm Au)

(290 000 toneladas con 7,9 ppm Au)

(250 000 toneladas con 7,9 ppm Au)

(658 000 toneladas con 1,7 ppm Au)

$3360 \mathrm{~kg}$ (108.000 onzas) Au de

109.000 toneladas mena.

(117 000 toneladas con 26,13 ppm Au)

$2706 \mathrm{~kg}$ (87.000 onzas) antes de 1960 .

(13 621.000 toneladas con 1,67 ppm Au)

(37,4 M toneladas; 1,63 ppm Au)

$87100 \mathrm{~kg}$ (2,8 millones onzas) $\mathrm{Au}$

$18700 \mathrm{~kg}$ (600 000) onzas Ag y

100000 toneladas $\mathrm{Zn}, 18000$

toneladas $\mathrm{Pb} 5000$ toneladas $\mathrm{Cu}$

(1,15 millones toneladas con 5,73 ppm Au)

(1,58 millones toneladas con 2,74 ppm Au,

15,1 ppm Ag, 0,84 \% Pb, 0,46\% Cu y 5,08 \% Zn)

$902 \mathrm{~kg}$ (29 000 onzas) Au y $1213 \mathrm{~kg}$

(39 000 onzas) $\mathrm{Ag}$ antes de 1954.

(2,6 millones toneladas con 8,6 ppm Au)

(544 000 toneladas con 6,51 ppm Au)

$31000 \mathrm{~kg}$ (1 millones onzas) Au de 226000 toneladas mena (1665-1727),

$31000 \mathrm{~kg}$ (1 millones onzas) Au de 900000 toneladas mena (1887-1907).

$2923 \mathrm{~kg}$ (93 971 onzas) Au y $176 \mathrm{~kg}$

(5 657 onzas) $\mathrm{Ag}(1905-1918)$

$2260 \mathrm{~kg}$ (72 500 onzas) Au (1914-1918)

(3,78 millones toneladas con 4,7 ppm Au)

$50000 \mathrm{~kg}$ (1,6 millones onzas) $\mathrm{Au}$

$124000 \mathrm{~kg}$ (4 millones onzas) Ag (1930-1987);

(1,7 millones toneladas con 4,2 ppm Au)

(9,3 M toneladas con 2,12 ppm $\mathrm{Au}$ )

\section{Referencias}

Chavez \& Saenz, 1974

Huber et al., 1987

Huber et al., 1987

Alan et al., 1992

Cooke, 1995

Stonehouse, 1976

Burn, 1969

Darce \& Tercero, 1992

Darce \& Tercero, 1992

Darce \&

Tercero, 1992

Darce \& Tercero, 1992

Ruiz-W., 1992

Woakes, 1923

Roberts \& Irving, 1957

Roberts \& Irving, 1957

Darce \& Tercero, 1992

Malone \&

Stoiber, 1987

Darce \& Tercero, 1992

Snyder, 1995 
Cuadro 4 (continuación)

Yacimientos de oro y plata tipo Bonanza

Montes del Aguacate,

Costa Rica (30)

San Juancito, Honduras (31)

San Sebastian,

El Salvador (32)

Topacio, Nicaragua (33)

Agua Fria,

Honduras (34)

El Transito,

Honduras (35)

La Unión,

Costa Rica (36)

Yuscaran, Honduras

(37)

San Pantaleón,

Guatemala (38)
$15000 \mathrm{~kg}$ (484 000 onzas) $\mathrm{Au}$

de 272000 toneladas mena antes de 1902

$23200 \mathrm{~kg}$ (746 000 onzas) Au y

$4 \mathrm{M} \mathrm{kg}$ (129000 000 onzas) Ag de

6,35 millones toneladas mena (1882-1954)

$23300 \mathrm{~kg}$ (750 000 onzas) $\mathrm{Au}$

hasta 1945

(507 000 toneladas con 6,51 ppm Au)

$2504 \mathrm{~kg}$ (80 500 onzas) Au de

180000 toneladas mena (1935-1943)

(125 000 toneladas con 6,2 ppm Au y

185000 toneladas con 8,5 ppm Au)

$15200 \mathrm{~kg}$ (490 000 onzas)

Au (1909-1960)

$15500 \mathrm{~kg}$ (500 000 onzas) Au y $886600 \mathrm{~kg}$ (28,5 millones onzas) Ag 1530-1821, 1880-1895, 1915-1923.

(525000 toneladas con 690 ppm Ag y

$5,0 \mathrm{ppm} \mathrm{Au})$

$622000 \mathrm{~kg}$ (20 millones onzas) Ag
Huber et al., 1987

Crespi, 1907; Chavez,1974

Carpenter, 1954

Roberts \& Irving, 1957

Wuensch, 1917

Darce \& Tercero, 1992

Svanholm, 1976

Roberts \& Irving, 1957

Cooke, 1974

United Nations

Revolving

Fund, 1988

* indica minas actualmente en producción

\section{YACIMIENTOS MINERALES Y TECTÓNICA}

Yacimientos de oro y cobre muestran una asociación espacial fuerte con la Depresión de Nicaragua. Esta estructura corre paralela a la fosa Centroamericana y mide entre sesenta a ochenta kilómetros de ancho. Fallas normales relacionadas con la fosa se extienden al sureste hasta Colombia y al noroeste hasta Guatemala.

La orientación de las estructuras mineralizadas dentro de la fosa con rumbo noroeste proporciona evidencia de movimiento paralelo al rumbo. Fallas de orientación este-oeste están mineralizadas en Santa Clara y Beta Vargas en Costa
Rica y en el Cerro Caballo de Panamá. Fallas norte-sur encajan mineralización aurífera a través de la franja minera de Veraguas (Panamá) y en Rio Chiquito (Costa Rica). Movimientos de tipo siniestral transcurrente son particularmente fuerte en el sur de Centroamérica donde el Bloque Panamá empuja hacia el norte sobre la placa Caribe.

Actividad de movimiento paralelo al rumbo no está restringido al sur de Centroamérica. Se hallan evidencias de actividad izquierdo-lateral a lo largo de fallas regionales con rumbo noroeste tan al norte como Honduras. La alteración en el prospecto del skarn de La Victoria, en la parte central de Honduras, exhibe varios kilómetros de desplazamiento lateral izquierdo. 
Cuadro 5

Yacimientos de oro epitermal (fuentes termales someras)

\section{Yacimiento \\ (Ubicación) \\ Remance*, \\ Panamá}

(39)

Río Chiquito,

Costa Rica

(40)

San Andres*, Honduras

(41)

Santa Clara,

Costa Rica (42)

Santa Rosa*,

Panamá (43)

Crucitas, Costa Rica (44)
Producción Pasada

(Reserva/Recurso)

$162 \mathrm{~kg}$ (5231 onzas) de 15500 toneladas

de mena (siglo 19), $840 \mathrm{~kg}$ (27 124 onzas)

de 70000 toneladas (1923-1932).

(250 000 toneladas con 5,5 ppm Au)

$110 \mathrm{~kg}$ (3523 onzas) Au y $890 \mathrm{~kg}$ (28 600 onzas)

$\mathrm{Ag}$ de 93500 toneladas de mena (1987-1989).

(1 millones toneladas con 5,62 ppm Au)

$2177 \mathrm{~kg}$ (70 000 onzas) Au de

363000 toneladas (1948-1954),

$706 \mathrm{~kg}$ (22 712 onzas) Au de

485371 toneladas (1983-1991).

(8,6 millones toneladas con 2,0 ppm Au)

$1244 \mathrm{~kg}$ (40 000 onzas) Au (1981-1989).

(4,5 millones toneladas con 1,6 ppm $\mathrm{Au}$ )

(6,5 millones toneladas con 1,7 ppm Au),

(22,7 millones toneladas con 1,4 ppm Au)

(58 millones toneladas con 1,2 ppm Au)
Referencias

Wleklinski, 1969

Nelson, 1992

Johnson et al., 1993

Notholt, 1987

White, 1993

Johnson et al., 1995

Northern Miner Nov. 27, 1995

* indica minas actualmente en producción

La extensión facilitó el emplazamiento de magmas someros. A lo largo de Centroamérica, domos e intrusivos exógenos fueron introducidos a lo largo de estructuras relacionadas con la fosa. Estos campos de domos están rodeados por mantos piroclásticos que encajan depósitos auríferos epitermales incluyendo a Crucitas, Santa Rosa, Remance, San Andrés y Río Chiquito. Vetas de bonanza y de stockworks se hallan en terreno más erosionado y se agrupan alrededor de

Cuadro 6

Yacimientos de sulfuros masivos y de alta sulfuración

\begin{tabular}{lll}
\hline $\begin{array}{l}\text { Yacimiento } \\
\text { (Ubicación) }\end{array}$ & $\begin{array}{l}\text { Producción Pasada } \\
\text { (Reserva/Recurso) }\end{array}$ & Referencias \\
$\begin{array}{l}\text { Cerro Quema, } \\
\text { Panama (8) }\end{array}$ & $\begin{array}{l}\text { con 10 millones toneladas } \\
\text { com Au) }\end{array}$ & $\begin{array}{l}\text { Torrey \& } \\
\text { Keenan, 1994 } \\
\text { Nelson, 1995 }\end{array}$ \\
$\begin{array}{l}\text { Oxec, } \\
\text { Guatemala (19) }\end{array}$ & $\begin{array}{l}\text { Petersen \& } 2,62 \% \text { Cu } \\
\text { Zantop, 1980 }\end{array}$ \\
\hline
\end{tabular}

centros de actividad intrusiva subvolcánica. Depósitos porfídicos en el sur de Centroamérica ocurren a lo largo de los márgenes de batolitos múltiples de composición intermedia pero también exhiben control por estructuras de orientación noroeste (p.ej.: Petaquilla y Cerro Colorado ).

\section{VETAS AURÍFERAS DE BONANZA}

La mayor parte de la producción aurífera en Centroamérica ha provenido de yacimientos tipo bonanza de alta ley y bajo tonelaje. Los depósitos de bonanza son vetas y enjambres modestas de relleno de fisuras encajadas por rocas volcánicas con alteración propilítica (Nelson, 1990). Las vetas tienen típicamente un buzamiento alto, son rellenadas por una ganga de cuarzo, calcita y adularia y son minadas para oro, plata y ocasionalmente metales básicos. El distrito Abangares en Costa Rica tiene una producción 
reportada de 936000 onzas de oro para el período de 1900 a 1913. La Unión y Montes del Aguacate, también en Costa Rica, cada una produjo como medio millón de onzas. Otros productores principales de oro en bonanzas incluyen $\mathrm{El} \mathrm{Li}$ món y minas Bonanza en Nicaragua, el distrito Caná en Panamá, San Sebastián en El Salvador y Yuscarán en Honduras.

\section{STOCKWORKS AURÍFEROS EN ROCAS MAÁRICAS}

Andesitas del arco insular inicial alteradas propilíticamente a través de Centroamérica fueron intruidas por complejos de tapones y flujos de domos de composición andesítica a riolítica. Estos complejos de intrusivos someros se agrupan en una inconformidad regional que separa a las andesitas inferiores con alteración propilítica de las rocas volcánicas sobreyacientes levemente alteradas y con la misma composición. La alteración regional a cuarzo-clorita-calcita es probablemente el resultado de la interacción con el agua del mar. La inconformidad marca la emergencia del arco insular del océano.

Domos exógenos están rodeados por mantos permeables de piroclastos y, en algunos distritos, por una secuencia distinctiva de sedimentos carbonosos y brechas epiclásticas en capas delgadas. En los márgenes de cráteres maáricos y en secuencias carbonosas/sedimentarias intravolcánicas se formaron depósitos de stockwork y oro diseminado. Vetas alimentadoras tienen típicamente orientación norte-sur o esteoeste en acuerdo con el movimiento transcurrente sobre fallas regionales con rumbo al Noroeste. Descubrimientos recientes sobrepasan el millón de onzas y representan un importante blanco para exploración. El potencial máximo, tal y como lo indica el yacimiento de Pueblo Viejo encajado en rocas maáricas en la República Dominicana, es de un orden de magintud mayor. Ejemplos de depósitos encajados en rocas maáricas incluyen los de Santa Rosa y Remance en Panamá y el Crucitas en Costa Rica. Santa Rosa fue descrito por Nelson (1990), Tippett \& Trever (1989) y White (1993).

\section{YACIMIENTOS PORFÍDICOS DE ORO-COBRE}

La mineralización de oro y cobre relacionada a pórfidos está asociada con intrusiones calco-alcalinas de edad paleocénica a pliocénica. La mayor parte ha sido emplazada en estratovolcanes que estaban activos antes del cierre del Istmo Centroamericano. En las provincias occidentales de Panamá, una cordillera alta expone plutones que varían en edad desde $35 \mathrm{Ma}$ (Petaquilla) hasta $6 \mathrm{Ma}$ (Cerro Colorado). La actividad intrusiva es significativamente más vieja en el oriente de Panamá y la península Azuero donde los plutones y la mineralización porfídica asociada varía en edad desde 65 Ma (Cerro Azul) hasta 49 Ma (Río Pito).

Todos los sistemas porfídicos estudiados hasta la fecha en la parte sur de Centroamérica están enriquecidos en oro así como en cobre. En algunos casos, el oro está alrededor de la mineralización de cobre (p.e. Cerro Colorado). En otros lugares el oro se halla sobreimpuesto o traslapa la mineralización de cobre (p.ej. Petaquilla, Río Pito, Cerro Chorcha). Un conducto brechoso y vetas en el distrito porfídico Caná de Panamá produjeron más de dos millones de onzas de oro de una mena de alta ley. Yacimientos porfídicos de cobre del sur de Centroamérica han sido descritos por Kesler et al. (1977) y Nelson (1994).

\section{YACIMIENTOS DE REEMPLAZAMIENTO Y DE SKARN}

Yacimientos de oro, molibdeno y cobre encajados en rocas de skarn se dan en el grupo de calizas Yojoa en Minas de Oro, Honduras. Johnson (1993) reporta que los skarns encierran un plutón granodiorítico y están compuestos por diópsido, magnetita y granate. Depósitos de skarn en el este de Nicaragua están encajados por una secuencia clástica sedimentaria con matríz carbonática cerca al contacto con plutones porfídicos de una granodiorita a diorita cretácica (Venable, 1994). El Yacimiento Siuna (La Luz) produjo más de un millón de onzas de oro (Plecash \& Hopper, 1963). 
El Mochito, la mina más grande en Honduras, es un depósito de reemplazamiento encajado por el miembro Atima de la caliza Yojoa. Desde 1948, la mina ha producido 11 millones de toneladas de mena con 750000 toneladas de zinc, 500000 toneladas de plomo, 300000 toneladas de cobre y más de 100 millones de onzas de plata (Johnson, 1993). La mena ocurre en chimeneas y como un manto a lo largo del contacto entre la caliza y la Formación Todos Santos subyaciente.

\section{CONCLUSIONES}

La mayor parte de los depósitos de oro y cobre en Centroamérica son encajados por rocas volcánicas del arco insular que empezaron a acumularse en el Cretácico tardío frente a la costa oeste de las Américas. Basaltos toleíticos bajos en potasio forman la parte basal del arco; rocas calcoalcalinas dominan ya para el período Mioceno Medio. Rocas volcánicas alcalinas típicamente son del Plioceno y más jóvenes, pero son poco reconocidas en porciones más antiguas de la estratigrafía volcánica.

La mayor parte de la producción histórica de oro ha venido de vetas de bonanza y conductos de brechas epitermales. Media docena de distritos de vetas bonanza en Centroamérica han producido más de un millón de onzas de oro. Varios descubrimientos auríferos recientes minables por metódos de cielo abierto también sobrepasan el millón de onzas. El yacimiento Santa Rosa en Panamá y el de Las Crucitas en Costa Rica se formaron en un ambiente epitermal somero. Rocas encajantes incluyen domos exógenos de composición basáltica a riolítica, brechas tobáceas que forman un manto piroclástico permeable, y delgados estratos de sedimentos carbonosos y brechas epiclásticas. En la República Dominicana, Pueblo Viejo contiene más de 570 toneladas de oro (20 millones de onzas) y proporciona documentación sobre el potencial superior de depósitos maáricos.

Yacimientos de cobre porfídico en el sur de Centroamérica son grandes y todos tienen mineralización aurífera asociada. Tanto Cerro
Colorado como Petaquilla en Panamá contienen recursos por encima del billón de toneladas. Sin embargo, el enriquecimiento secundario es débil o ausente, la ley del cobre es típicamente menor al uno porciento y el oro aumenta tan solo marginalmente el valor de la mena. Depósitos de skarn y de reemplazamiento hallados en los bloques Chortis y Maya incluyen yacimientos de hierro, cobre-oro, y plomo-zinc-plata. El depósito de skarn Siuna en Nicaragua produjo mas de un millón de onzas de oro mientras que depósitos de reemplazamiento en la mina Mochito han producido cobre, plomo, zinc y plata desde 1948. Cerro Quema, el primer depósito de alta sulfuración que fué descubierto en Centroamérica, se dice contener diez millones de toneladas con una ley de 1,26 ppm de oro.

Nuevos descubrimientos de cobre y oro pueden esperarse a lo largo de fallas regionales relacionadas a la fosa, particularmente dentro de un arco insular intraoceánico primitivo. Areas recomendadas para exploración en el sur de Centroamérica incluyen la faja porfídica de Costa Rica donde ésta se proyecta a través de la frontera hasta Panamá, dentro de campos de domos exógenos a lo largo de los márgenes de la Depresión de Nicaragua, y dentro de complejos de rocas volcánicas alcalinas generalmente poco reconocidas en la sección volcánica terciaria. Áreas recomendadas para exploración en el núcleo de Centroamérica incluyen las margenes de las zonas de fosa de rumbo norte-sur, la margen norte de la placa Caribe, y las margenes de la Depresión de Nicaragua.

\section{REFERENCIAS}

ADRIAN RESOURCES LTD., 1994: Preliminary feasibility study completed - Total property resources exceed 16 billion tonnes.- Adrian Resources Ltd. press release, August 19, 1994.

ALAN, M.A., QUESADA, J.D., SEAWARD, M. \& HUTTON, D., 1992: Economic geology of the Bellavista gold deposit, Costa Rica. - Soc. for Mining, Metallurgy, and Exploration, Inc., preprint number: 92-132. 
ALVARADO, G.E., KUSSMAUL, S., CHIESA, S., GILLOT, P.-Y., APPEL, H., WÖRNER, G. \& RUNDLE, C.1994: Resumen cronostratigrafico de las rocas igneas de Costa Rica basado en dataciones radiometricas. - J.South American Earth Sci.6(3): 151-168.

APPEL, H., WÖRNER, G., ALVARADO, G., RUNDLE, C. \& KUSSMAUL, S., 1994: Age relations in igneous rocks from Costa Rica. - Profil, 7: 63-69.

ARLO RESOURCES, 1995: Summary of Panamanian Projects. - [informe interno].

BONIS, S., BOHNENBERGER, H. \& DENGO, G., 1970: Mapa geológico de la República de Guatemala (1:500 000). - Instituto Geográfico Nacional, Guatemala.

BURN, R.G., 1969: The Pis Pis gold mining district of northeast Nicaragua. - Mining Mag. 120: 169-175.

CARPENTER, R.H., 1954: Geology and ore deposits of the Rosario mining district and the San Juancito Mountains, Honduras. Geol. Soc.America Bull. 65: 23-38.

CASE, J.E. \& HOLCOMBE, T.L., 1980: Geologic-tectonic map of the Caribbean Region (1:2 500000 scale). - U. S. Geological Survey Miscellaneous Investigations Series Map I-1865.

CHÁVEZ, R., 1974: The Aguacate mines of Costa Rica - Geology and Appraisal. - Inf. técnicos y notas geol., 57, Dir. de Geol., Minas y Petróleo, 49 págs.

CHÁVES, R. \& SÁENZ, R., 1974: Geología de la Cordillera de Tilarán. - 49 págs. Ministerio de Economía, Industria y Comercio, Dir. de Geol., Minas y Petróleo.

CLARK, G.H., 1982: The Cerro Colorado porphyry copper project. - 40 págs. RTZ
Group Exploration Conference, Johannesburg [informe interno].

COOKE, B.J., 1995: Geological resources double to 2 million ounces gold at Bellavista Gold Project, Costa Rica. - Canarc Resource Corp. News Release, October 3, 1995.

COOKE, H.R., 1974: La Union gold district, Montes de Oro, Puntarenas, Costa Rica. 28 págs. Cooke, Everett and Ass. Inc. [informe interno No. 151 E].

CRESPI, R.A., 1907: Geology and development of Aguacate mines, Costa Rica. - The Mining World, November 9: 847-848.

DARCE, M., 1989: Mineralogical alteration patterns, chemical mobility and origin of the La Libertad gold deposit, Nicaragua. - 103 págs. University of Stockholm [Tesis $\mathrm{PhD}$.].

DARCE, M., 1990: Mineralogic alteration patterns in volcanic rocks of the La Libertad gold mining district and its surroundings, Nicaragua. - Economic Geol. 85: 10591071.

DARCE, M. \& TERCERO G., A., 1992: Estado de reservas auriferas del sector minero Nicaraguense al 1 de Enero de 1992. - 11 págs. Direccion General de Geologia, INMINE Central.

DENGO, G., LEVY, E., BOHNENBERGER, O. \& CABALLEROS, R., 1969: Mapa metalogenético de América Central (1:2 000 000 scale). - Instituto Centroamericano de Investigacion y Technologia Industrial (ICAITI).

DONNELLY, T.W., HORNE, G.S., FINCH, R.C. \& LOPEZ-RAMOS, E., 1990: Northern Central America; the Maya and Chortis blocks. - En: DENGO, G. \& CASE, J.E. (eds.): The Caribbean Region. - Boulder, 
Colorado, Geol. Soc. America, The Geology of North America, H: 37-76.

ESCALANTE, G., 1990: The geology of southern Central America and western Columbia. - En: DENGO, G. \& CASE, J.E. (eds.): The Caribbean Region: Boulder, Colorado, Geol. Soc. America, The Geology of North America, H: 201-230.

FISCHER-WATT, 1992: October 29 report to the shareholders. - 2 págs. Fischer-Watt Gold Company, Inc.

FERENCIC, A., 1971: Metallogenic provinces and epochs in Southern Central America. Mineral. Deposita, 6: 77-88.

HARJU, H.O., 1979: Exploration of Eximbal's nickel laterite deposits in Guatemala. - En: EVANS, D.J.L. (ed.): International laterite symposium. - Soc. Mining Engineers: 57-84.

HORNE, G.S., CLARK, G.S. \& PUSHKAR, P., 1976: Pre-Cretaceous rocks of northwestern Honduras: Basement terrane in Sierra de Omoa. - Amer. Ass.Petrol. Geol. 60: 566-583.

HUBER, D.F., PAGE, N.J., HOWE, S.S. \& SOLANO, C., 1987: Mines, prospects and mineral occurrences in Costa Rica. - U. S. Geol. Survey Miscellaneous Investigations Series Map I-1865: 18-33.

JOHNSON, M.J., 1993: Mining and mineral deposits of Honduras and El Salvador. - En: Randol at Acapulco '93 - Conference Proceedings. - Randol International Ltd.: 87-100.

JOHNSON, M.D., HILLEMEYER, F.L.\& BYBEE, R.W., 1993: Geology and mineralization at the San Andres mine, Honduras. Soc. Mining, Metallurgy, and Exploration, Inc., preprint number 93-206, 24 págs.

JOHNSON, M.D., BIKERMAN, D. \& RUSSELL, M., 1995: Function of contemporary mode- lling in mine development at Mina Santa Rosa, Veraguas Province, Panama. - Soc. Mining Engineers, Preprint Series.

KESLER, S.E., 1978: Metallogenesis of the Caribbean region. - J. Geol. Soc. London, 135: 429-441.

KESLER, S.E., SUTTER, J.F., ISSIGONIS, M.J., JONES, L.M. \& WALKER, R.L., 1977: Evolution of porphyry copper mineralization in an oceanic island arc, Panama. - Econ. Geol., 72: 1142-1153.

KESLER, S.E., LEVY, E. \& MARTIN, F.C., 1990: Metallogenic evolution of the $\mathrm{Ca}-$ ribbean region. - En: DENGO, G. \& CASE, J.E. (eds.): The Caribbean Region. Geol. Soc. America, The Geology of North America, H: 459-482.

KOZUCH, M.J., 1990: Mapa geológico de Honduras (1:500 000). - Inst. Geografico Nacional, Honduras.

KUSSMAUL, S., TOURNON, J. \& ALVARADO, G.E., 1991: Evolución de las rocas plutónicas y volcánicas subalkalinas del Neogeno y Cuarternario de Costa Rica. Memorias Simposio sobre Magmatismo Andino y su marco Tectonico, Manizales, Colombia. 1: 23-44.

LEVY, E., 1970: La metalogénesis en América Central. - Publicaciones Geológicas del Instituto Centroamericano de Investigacion y Tecnologia Industrial, 3: 17-57.

MACKEVETT, E.M., Jr., 1980: Geology and exploration of the Matama II (Nari) project area, Costa Rica, C.A. - 26 págs. [informe interno Mapco].

MALONE, G.B. \& STOIBER, R.E., 1987: Caldera-related gold mineralization of the El Limon mining district, western Nicaragua. -.J. Volcan. Geothermal Res. 33: 217-222. 
MANN, P. \& CORRIGAN, J., 1990: Model for late Neogene deformation in Panama. Geology. 18: 558-562.

MOE, K., 1992: Canadian firm steps up gold exploration in Honduras. - Mining Record, 103(17): 1 .

NELSON, C.E., 1990: Bulk mineable epithermal precious metal deposits of the Circum-Caribbean region. - En: Hausen, D.M., Halbe, D.N., Petersen, E.U. \& Tafuri, W.J. (eds.): Proceedings of the Gold '90 Symposium. - Soc. Mining, Metallurgy and Exploration, Inc.: 149-161.

NELSON, C.E., 1992: Gold mineralization in maar craters - two examples from southern Central America. - En: Randol at Minexpo '92 -Proceedings. - Randol International Ltd., 55-58

NELSON, C.E., 1995: Porphyry copper deposits of southern Central America. - Arizona Geol. Soc. Digest, 20: 553-565.

NORTHERN MINER, 1995: Placer Dome boosts budget to explore and develop international gold properties. - Northern Miner, Nov. 27, 1995, pág. C12.

NOTHOLT, A., 1987: Central America. - Mining Annual Review: 326--328.

PETERSEN, E.U. \& ZANTOP, H., 1980: The Oxec deposit, Guatemala; an ophiolite copper occurrence. - Econ. Geol., 59: 753-767.

PINDELL, J.L. \& BARRETT, S.F., 1990: Geological evolution of the Caribbean region; a plate-tectonic perspective. - En DENGO, G. \& CASE, J.E. (eds.): The Caribbean Region: Boulder, Colorado, Geol. Soc. America, The Geology of North America, H: 405-432.
PLECASH, J. \& HOPPER, R.V., 1963: Operations at La Luz Mines and Rosita Mines, Nicaragua, Central America. - Canadian Mining and Metallurgical Bull.: 624-641

ROBERTS, R.J. \& IRVING, E.M., 1957: Mineral deposits of Central America. - 205 págs. U. S.Geol. Survey Bull. 1034.

RUIZ-WILLIAMS, H., 1992: Executive summary - 6 págs.Cana Mining District, Panama [informe interno]

SNYDER, H.R., 1995: Greenstone Resources Third Quarter Report. - November 24, 1995.

STONEHOUSE, J.M., 1976: Movement of mineralizing fluids, Bonanza mining district, Nicaragua. - 64 págs. Dartmouth College, New Hampshire [Tesis MSc.].

SVANHOLM, J., 1976: Gold in Honduras - Where to find it. - Publ. geol. del Instituto Centroamericano de Investigación y Technología Industrial (ICAITI), V, Guatemala: 206236.

TIPPETT, M.C. \& TREVER, P.F., 1989: The isthmus of Panama - A forgotten El Dorado. - Soc. Mining Engineers, Preprint number 89(56), 4 págs.

TORREY, C. \& KEENAN, J., 1994: The Cerro Quema Project, Panama. - 13 págs. [unpublished fieldtrip guidebook].

TOURNON, J. \& ALVARADO, G., 1997: Carte Geologique du Costa Rica / Mapa geológico de Costa Rica. - 79 págs. Ed. Tecnológica de Costa Rica, Cartago.

\section{UNITED NATIONS REVOLVING FUND FOR NATURAL RESOURCES EVALUATION, 1981: Exploración para cobre y oro en Río Pito, Panamá. - UNRFNRE final report to the government of the Republic of Panama,}


DP/PAN/NR/77-001, 150 págs.

UNITED NATIONS REVOLVING FUND FOR NATURAL RESOURCES EVALUATION, 1988: Exploration for precious and base metal in Honduras. - Project HON/NR/83/001, 1987 Annual Report, 79 págs.

VENABLE, M.E., 1994: A geologic, tectonic, and metallogenic evaluation of the Siuna Terrane. - 154 págs. Univ. of Arizona [Tesis $\mathrm{PhD}$.].

WHITE, D.C., 1993: Geology and Mineralization of the Santa Rosa gold deposits, Panama. - 5 págs. Soc. Mining Engineers, Preprint Series,
WILLIAMS, R.L. \& SANCHEZ, J.K., 1972: Mapa geológico de Nicaragua Occidental (1:250 000. - División de Geología de Catastro, Nicaragua.

WLEKLINSKI, S., 1969: Gold deposits of northern Veraguas. - 113 págs. United Nations Development Program, Mineral Survey of the Azuero Area, 1.

WOAKES, E.R., 1923: The Darien gold mine, Panama. - Mining Mag.: 270-278.

WUENSCH, C.F., 1917: Geology of the San Sebastian mine, Salvador. - Mining Science Press: 345-350. 